\title{
Unknown syndrome: peculiar face, severe hypodontia of permanent teeth, and precocious choroid calcifications
}

\author{
Rosanna Pallotta, Paola Fusilli
}

\begin{abstract}
We describe a mother and her twin daughters affected with severe hypodontia of the permanent teeth, precocious calcification of the choroid plexus, and minor digital anomalies. The presence of inner telecanthus, broad and flattened nasal bridge, mild ocular proptosis, small nose with anteverted nostrils, and slight microretrognathia gives them an unusual appearance. All three affected persons are of normal intelligence.

$(\Im$ Med Genet 1998;35:435-437)
\end{abstract}

Keywords: oligodontia; persistent deciduous teeth choroid plexus calcifications

Both hypodontia and anodontia are well known disorders. Complete anodontia is very rare; according to Bartsocas, ${ }^{1}$ Pyrrhus (318-272 BC), King of Epirus, may have been affected. It is inherited as an autosomal recessive trait. ${ }^{2}$

Witkop ${ }^{3}$ suggested that the trait of small, peg shaped, or missing maxillary lateral incisors not associated with a syndrome is best explained as an autosomal dominant trait modified by a polygenic component, and that agenesis of succedaneous teeth is a recessive trait that results from the homozygous state of this gene. ${ }^{4}$ Dolamore $^{5}$ described a case of persistent deciduous canines with absence of permanent successors. Gorlin (personal communication) pointed out that about a third of the general population lack one or more of the third molars (wisdom teeth), the teeth most often missing, followed by the premolars. Anodontia and

Revised version accept publication

11 November 1997

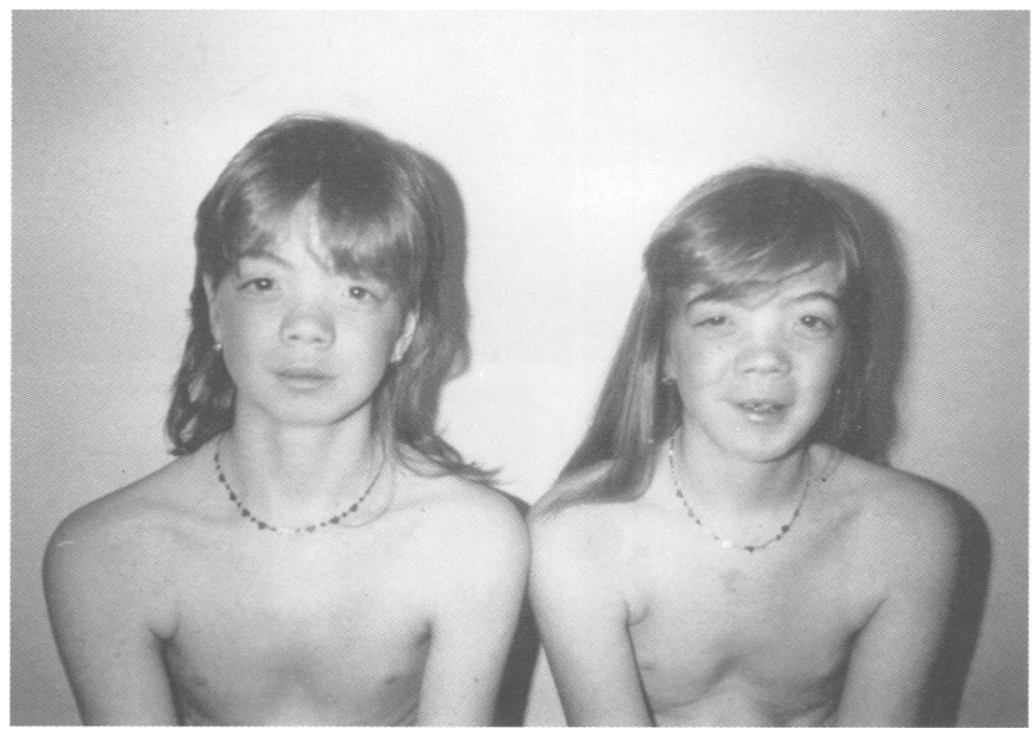

Figure 1 Front view of the girls at 12 years of age. hypodontia are often the expression of complex situations involving defective formation of structures of ectodermal origin.

We describe a family who have, to our knowledge, a completely new condition.

\section{Case report}

We report a family which includes a 39 year old affected mother and her 20 year old twin daughters. At the time of conception their father was 24 years old. They are of southern Italian origin, their mother, the third of five children (three males and two females), coming from Sicily and their father, the second of three brothers, from Abruzzo; the parents are not related. The family history on both sides was negative for miscarriages, malformations, and genetic or metabolic disease. The girls were born at 31 weeks of gestation and were registered as monochorionic identical twins. Their growth was appropriate for age (90th centile), they were negative for transplacental infections (TORCH), and their psychomotor development was absolutely normal. The blood group of all three patients was $\mathrm{O}$ $\mathrm{Rh}(\mathrm{D})+$. They came to our attention at the age of 12 years through genetic counselling. Their early medical history, like that of their mother, was unremarkable; growth parameters were still around the 90th centile and their intelligence was well above average.

The twins' faces showed very similar characteristics to that of their mother (figs 1,2 , and 3), although more accentuated, and included inner telecanthus (ICD >97th centile, IPD >95th centile, $O C D>75$ th centile), downward slanting palpebral fissures, mild ocular proptosis, flattened, wide nasal bridge, a small nose with forward slanting nostrils, slight microretrognathia, and severe oligodontia of the permanent dentition, the upper central incisors $(11,21)$ and first molars being present $(16,26,36,46)$ with persistence of the remaining deciduous teeth. The resulting dental formula was:

\begin{tabular}{llllll|llllll}
16 & & & & & 11 & 21 & & & & & 26 \\
& 55 & 54 & 53 & 52 & & & 62 & 63 & 64 & 65 & \\
\hline 46 & 85 & 84 & 83 & 82 & 81 & 71 & 72 & 73 & 74 & 75 & \\
& & & & & & & & & & & 36
\end{tabular}

Furthermore, questionable loss of the interdental vertical dimension, full lips, pectus carinatum, clinodactyly of the fifth finger, and hypoplastic distal phalanx of the second toe were observed. The mother had a right single transverse palmar crease.

In all three family members, cranial radiographs showed two bilateral and symmetrical, 


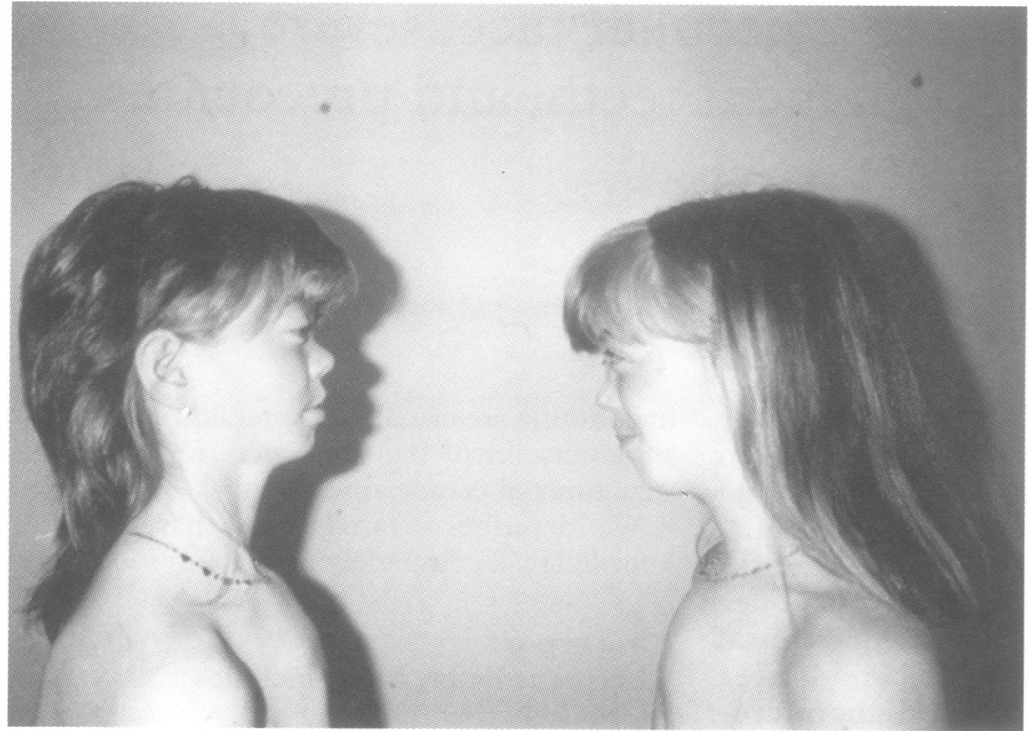

Figure 2 Profile of the same girls.

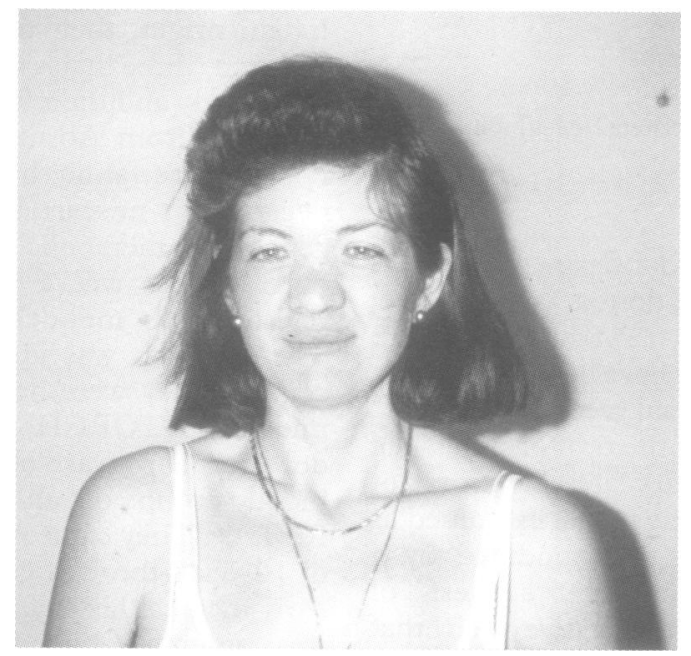

Figure 3 The mother.

rounded, calcified masses (fig 4, top row) apparently localised at the level of the basal ganglia, hypoplastic greater wings of the sphenoid, narrowed, cribriform plate of the eth- moid bone, fused clinoid processes, hypoplastic nasal septum, and agenesis of all permanent teeth, except for the upper middle incisors and first upper and lower molars. These findings were all confirmed three months later on CT scan, except for the large calcifications, which were situated at the level of the choroid glomus (fig 4, bottom row) but not accompanied by signs of intracranial hypertension. The metacarpophalangeal profile (MPP) showed some shortening of all the second phalanges, more accentuated in the fifth finger. $X$ rays also confirmed the shortening of the distal phalanx of the second toe. The remaining skeletal, ophthalmological, and cardiac examinations were normal.

Dermatoglyphic analysis showed: (mother) right: Wd, Wd, Wd, W, W; palmar a-b ridge count $(a-b)=40 ; a \hat{t d}=40^{\circ}$; total finger ridge count $($ TFRC $)=148$; mainline formula $=$ 11.9.7.5”.13" t, left: Wd, Lr, Wd, Wd, Lr; $a-b=43 ; \quad a t d=40^{\circ} ; \quad$ TFRC $=130 ;$ mainline formula $=9.7 .5^{\prime} .5^{\prime} \cdot 13^{\prime}$-t. (1 st girl) right: Lu, $\mathrm{Lr}, \mathrm{Lu}, \mathrm{W}, \mathrm{Wd}$; $\mathrm{a}-\mathrm{b}=44$; $\mathrm{atd}=47^{\circ}$; TFRC $=97$; mainline formula $=11$. 9.7.5'.13'-t, left: $\mathrm{W}, \mathrm{Lr}$, Lr, W, W; $a-b=37 ; a \hat{t d}=43^{\circ}$; TFRC $=107$; mainline formula $=9.9 .5^{\prime} \cdot 5^{\prime} \cdot 13^{\prime}-\mathrm{t}$. (2nd girl) right: Wd, Lr, A, Wd, Wd; $a-b=40$; atd $=44^{\circ}$; TFRC =97; mainline formula $=11$. 9. 7. 5'. 13'-t, left: Wd, Lr, Lr, W, Wd; $a-b=43$; atd $=44^{\circ}$; TFRC $=93$; mainline formula $=11$. 9 . 7. 5'. 13'-t.

Direct counting of the patients' sweat pores in different areas of the palm and fingers was normal. The karyotype was normal. In spite of the absence of any neurological or bone density abnormality, the intracerebral calcifications led us to perform several laboratory tests, including calcium and parathormone level, which all proved to be normal.

We followed up the family for over eight years. During this period nothing of particular clinical interest was observed. The problem of the dental anomalies was solved by extracting the deciduous teeth and applying a permanent prosthesis in the mother and by applying a
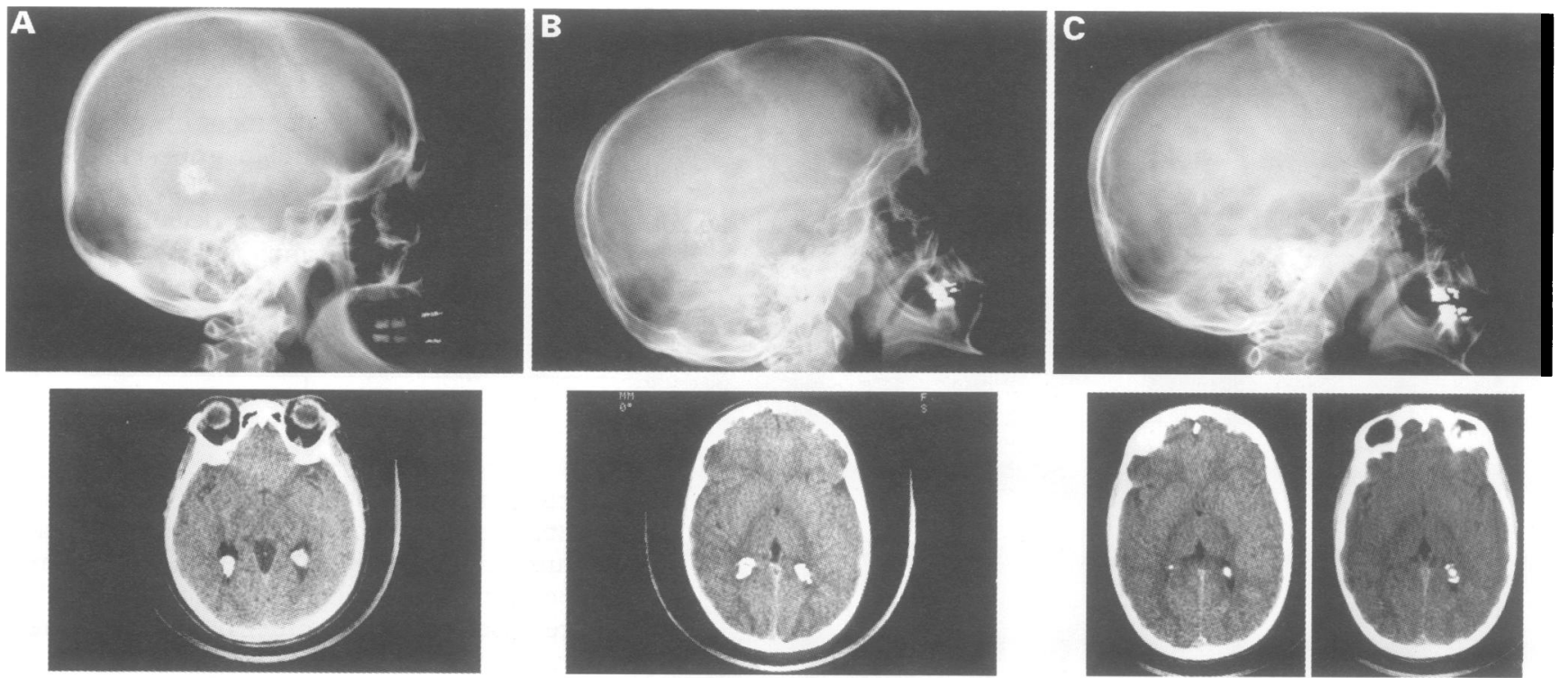

Figure 4 Calcifications of the choroid glomus in $(A)$ the mother, $(B)$ the first girl, and $(C)$ the second girl. Top row: lateral skull radiographs, bottom row: cranial CT scan. 
Table 1 Phenotype of the family: note that the expression of the gene defect is identical in all three subjects

\begin{tabular}{llll}
\hline & Mother & 1st girl & 2nd girl \\
\hline Inner telecanthus & + & + & + \\
Downward slanting palpebral fissures & + & + & + \\
Mild ocular proptosis & + & + & + \\
Flattened, wide nasal bridge & + & + & + \\
Small nose with forward slanting nostrils & + & + & + \\
Hypoplastic maxilla & + & + & + \\
Slight microretrognathia & \pm & + & + \\
Persistence of deciduous teeth & + & + & + \\
Permanent teeth: 11, 21, 16, 26, 36, 46 & + & + & + \\
Full lips & + & + & + \\
Pectus carinatum & + & + & + \\
Clinodactyly of the 5th finger & + & + & + \\
Hypoplastic distal 2nd phalanx of the foot & + & + & + \\
Hypoplastic wings of the sphenoid & + & + & + \\
Hypoplastic nasal septum & + & + & + \\
Agenesis of permanent teeth & + & + & + \\
High total finger ridge count & + & + & + \\
Bilateral choroid plexus calcifications & + & + & + \\
Mental retardation & - & - & -
\end{tabular}

conservative prosthesis to the deciduous teeth in the daughters. The growth parameters have remained on the 90th centile. School results confirmed the high intelligence level of the twins.

\section{Discussion}

Although the twins had been referred to at birth as monochorionic and identical, we also performed the following: dermatoglyphic analysis, ${ }^{6}$ blood groups, the Goldsmith twin similarity questionnaire, ${ }^{7}$ and the ponderal index (Pondex). ${ }^{8}$ The conclusions drawn from all the results were consistent with monozygosity.

The patients reported here showed not only dental abnormalities (the permanent teeth consisting merely of the upper central incisors and first molars with persistence of the remaining deciduous teeth), but also ocular proptosis resulting from malformations of the sphenoid and ethmoid and hypoplasia of the nasal septum. These anomalies, together with the inner telecanthus and full lips, give them a quaint, flat profile. The bone anomalies of the limbs are not typical, consisting of clinodactyly of the fifth finger, hypoplasia of the terminal phalanx of the second toe, and shortening of the second phalanx of the fingers at the MPP.

The total finger ridge count in the mother was 283 , in the first twin 204, and in the second 204, all high values compared to the average 143.17 of a female population in southern Italy. ${ }^{9}$

As shown in table 1, the expression of the gene defect is identical in all three subjects. The bilateral and symmetrical calcifications of the choroid glomus were very interesting. The latter were already evident when the girls were 12 years old and, judging by their size, which was more or less the same in the daughters and mother, were presumably present long before the age of 10 years; they were bilateral and symmetrical.

Similar calcifications may also be found in metabolic diseases, such as pseudohypoparathyroidism. ${ }^{10}$ Although the cyclic AMP after infusion of exogenous parathormone in serum was not measured because of the unavailability of this hormone, the suspected hypoparathyroidism or pseudohypoparathyroidism was excluded by the absence of biological or clinical signs, including the skeletal anomalies.

In the absence of any clinical pathological signs, the calcifications of the choroid glomus in all three patients may be considered physiological. Calcifications with similar characteristics in the first decade of life have either not been found at all ${ }^{11}$ or very rarely, in $0.5 \% .^{12}$ It is well known that although these formations can occur as early as the first three years of life, they are very uncommon in patients under the age of $10 .{ }^{12}$

It is an established fact that familial calcifications of the choroid plexus are present in some

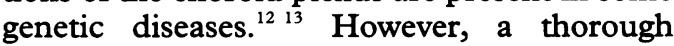
review, using computerised dysmorphology databases (both London and POSSUM), did not show any association of choroid glomus calcifications with hypodontia or facial anomalies or both that was similar to the features in our family.

To our knowledge, oligodontia with persistence of the decidous teeth and intracerebral calcifications has so far only been reported once, ${ }^{14}$ in two daughters of a consanguineous marriage showing the typical onion skin phenomenon, pathognomonic of lipoid proteinosis. The condition presented here is clearly different.

Thus, in spite of follow up lasting over eight years and in the absence of other similar cases, only a familial factor or possibly a new malformation syndrome, clearly of autosomal dominant inheritance, can be proposed.

We are grateful to Professor Francesco Schioppa (Department of Biomedical Sciences, University of Chieti, Italy), who helped in assessing monozygosity by statistical analysis, and to Dr Leda Dalprà (Department of Biology and Genetics for Medical Sciences, University of Milan, Italy), who performed the karyotyping of the patients.

1 Bartsocas CS. Complete absence of the permanent dentition: an autosomal recessive disorder. Am $\mathcal{f}$ Med Genet dentition: an auto:

2 Gorlin RJ. Total absence of the permanent dentition: an autosomal recessive disorder. Am f Hum Genet 1979;31:72A.

3 Witkop GJ Jr. Studies of intrinsic disease in isolates with observations on penetrance and expressivity of uncertain anatomical traits. In: Bruzansky S, eds. Congenital anomalies of the face and associated structures. Springfield, IL: C C Thomas, 1961:291-368.

4 Witkop GJ Jr. Agenesis of succedaneous teeth: an expression of the homozygous state of the gene of the pegged or missing maxillary lateral incisor trait. Am $\mathcal{Y}$ Med Genet 1987;26: 431-6.

5 Dolamore WH. Absent canines. Br Dent $\mathcal{F}$ 1925;46:5-8.

6 Reed T, Sprague FR, Kang KW, Nance WE, Christian JC. Genetic analysis of dermatoglyphic pattern in twins. Hum Hered 1975;25:263-75.

7 Spitz E, Moutier R, Reed T, et al. Comparative diagnoses of twin zygosity by SSLP variant analysis, questionnaire, and twin zygosity by SSLP variant analysis, questionnaire,

8 Likken DT. The diagnosis of zygosity in twins. Behav Genet 1978;8:437-73

9 Pallotta R, Carlone G, Petrucci A, Chiarelli F. Dermatoglyphics in von Recklinghausen neurofibromatosis. $\mathrm{Am} \mathfrak{f}$ Med Genet 1989;34:233-6.

10 Smit L, van-Wiyk RM, Rico RE, Sitalsing AD. Intracerebra bilateral symmetrical calcifications, demonstrated in a patient with pseudohypoparathyroidism. Clin Neurol Neurosurg 1988;90:145-50.

11 Kwak R, Takeuchi F, Ito S, Kadoya S. Intracranial physiological calcification on computed tomography. Part ventricles. No To Shinkei 1988;40:707-11.

12 Modic MT, Weinstein MA, Rothner AD, Erenberg G, Duchesneau PM, Kaufman B. Calcification of the choroid plexus visualized by computed tomography. Radiology 1980;135:369-72.

13 Singh B, Jamil A, Al-Shahwan SA, Sharif H, Al-Deeb SM, Biary N. Choroido-cerebral calcification syndrome with retardation. Neurology 1993;43:2387-9.

14 Oezarmagan G, Baykal C, Gursoy EO, Yilmazer S, Oezarmagan G, Baykal C, Gursoy EO, Yilmazer S,
Buyukbabani N, Coban O. Lipoid-proteinose bei zwei Schwestern. Hautarzt 1993;44:315-18. 